\title{
Traduire
}

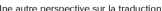

Revue française de la traduction

$221 \mid 2009$

Voies de l'interprétation

\section{Les différents types d'inférence dans la compréhension orale du japonais}

Franck Tiret

\section{(2) OpenEdition}

1 Journals

Édition électronique

URL : http://journals.openedition.org/traduire/342

DOI : $10.4000 /$ traduire.342

ISSN : 2272-9992

Éditeur

Société française des traducteurs

Édition imprimée

Date de publication : 15 décembre 2009

Pagination : 19-32

ISSN : 0395-773X

\section{Référence électronique}

Franck Tiret, « Les différents types d'inférence dans la compréhension orale du japonais », Traduire [En ligne], 221 | 2009, mis en ligne le 12 novembre 2013, consulté le 25 novembre 2020. URL : http:// journals.openedition.org/traduire/342 ; DOI : https://doi.org/10.4000/traduire.342 


\section{Les différents types d'inférence dans la compréhension orale du japonais}

\section{Franck Tiret}

La maîtrise du japonais pour les Occidentaux en général et pour les Français en particulier revêt des aspects différents selon les niveaux de langue visés et les objectifs assignés à cette connaissance linguistique. Ainsi, il n'est pas rare de rencontrer des traducteurs occidentaux travaillant depuis le japonais qui ont acquis un excellent niveau de lecture et de compréhension grâce à de solides études universitaires agrémentées d'un séjour plus ou moins long au Japon. L'enseignement universitaire étant surtout axé sur le travail de l'écrit, il n'est pas étonnant de constater que les étudiants qui ont suivi cette voie se sentent particulièrement à l'aise dans les exercices centrés sur la lecture et la traduction. Même si l'expression orale n'est pas pour autant laissée pour compte, le niveau généralement atteint dans ce domaine est souvent bien en deçà de celui atteint à l'écrit, ce qui incite les étudiants à faire des séjours dans le pays dans le but de parfaire leur expression orale ainsi que leur compréhension à l'écoute. Dans ces conditions, il est fréquent de rencontrer des personnes ayant atteint un niveau très correct en japonais leur permettant de s'exprimer couramment dans la vie quotidienne et dans la vie professionnelle, voire d'être très compétentes dans des domaines extrêmement spécialisés.

Cependant, force est de constater, comme le faisait déjà Daniel Gile en 1988, que le nombre d'interprètes occidentaux de liaison ou de conférence travaillant depuis le japonais est toujours très limité et en tout cas très nettement inférieur au nombre d'interprètes japonais travaillant depuis nos langues européennes. Les raisons qui peuvent expliquer un tel déséquilibre sont forcément d'ordre divers (linguistique, économique, culturel, etc.), mais sur le plan linguistique, la raison la plus fréquemment évoquée est celle des difficultés rencontrées à l'écoute. Pour un apprenant maîtrisant parfaitement la grammaire et la syntaxe japonaises, la compréhension d'interventions orales d'un niveau soutenu reste souvent une difficulté majeure due au grand nombre de mots inconnus ou non reconnus dans le discours en langue source (Gile, 1988). Ces difficultés semblent suffisamment dissuasives pour détourner des étudiants brillants des formations des écoles d'interprète comme celle de l'ESIT qui ne forment qu'aux techniques de l'interprétation, considérant les compétences linguistiques de leurs étudiants comme acquises. Nous sommes donc en présence d'une "zone de non-enseignement " pour le 
candidat à ce type de formation qui n'aura d'autre recours que l'auto-apprentissage ou l'autoperfectionnement, que la disponibilité des sources audio sur internet a heureusement rendu aujourd'hui plus facile.

Dans le but d'apporter quelques pistes de réflexion concernant ce problème, il m'a paru opportun, à la lumière de mon expérience professionnelle d'interprète, d'étudier les mécanismes mis en œuvre dans le processus de la compréhension orale du japonais pour un Français, et plus particulièrement sur les phénomènes d'inférence qui sont les mécanismes qui permettent de déduire des informations sur la signification d'un item lexical (défini ici comme un mot non grammatical appartenant au lexique d'une langue) non connu de l'auditeur français japonisant. Afin d'apporter quelques éléments quantitatifs destinés à étayer mes observations, j'ai réalisé une expérience portant sur la compréhension d'une dizaine d'émissions radiophoniques. II n'échappera pas au lecteur que la modestie des moyens mis en œuvre incite à la plus grande prudence quant à l'interprétation des résultats obtenus, l'objectif de cet article étant plutôt de lancer des pistes de recherche que d'apporter des réponses définitives.

\section{Quelques éléments de linguistique japonaise}

Bien que mon propos porte sur la compréhension des sources orales de japonais, nous allons voir, tout au long de cet article, que les relations entre l'oral et l'écrit y jouent un rôle déterminant, ce qui m'amène à donner quelques précisions sur le système de l'écrit. Le japonais contemporain est composé de quatre catégories de signes différents : les hiragana, les katakana, les kanji et nos lettres romaines. Les hiragana sont un système syllabique d'une cinquantaine de signes qui permettent de retranscrire la langue japonaise de manière phonétique. Ce sont des signes graphiquement assez arrondis qui sont utilisés pour les mots d'origine japonaise (comme le mot hanashi はなし, " la discussion ”) par opposition aux katakana, plus anguleux, qui sont leurs équivalents destinés à retranscrire les mots d'origine étrangère (comme le motトークショー tôkushô pour le mot anglais talk-show), leurs abréviations ainsi que certaines onomatopées. Parallèlement à ces syllabaires, sont utilisés des " idéogrammes " d'origine chinoise appelés kanji. Présents au Japon depuis des temps ancestraux, c'est l'arrivée du bouddhisme au vle siècle qui voit l'accélération de leur introduction. Chaque kanji représente généralement un concept et peut être associé à d'autres pour former des concepts plus élaborés ; par exemple en associant le kanji 会 kai, "la rencontre ", et le kanji 話 wa, "la parole ", on obtient le mot 会話 kaiwa, qui signifie "la conversation ". Si la plupart des associations de kanji peuvent paraître répondre à une logique évidente, voire universelle, ce n'est pas toujours le cas pour l'apprenant occidental; dans le mot 船長 senchô, nous avons en premier un kanji exprimant le concept de "bateau " suivi de celui qui exprime le concept de "longueur ". Contrairement à ce que l'on pourrait penser, ce mot n'exprime pas "la longueur du bateau " mais "le capitaine du bateau ". Ici le deuxième kanji désigne la 
personne qui a le plus d'ancienneté sur le bateau, donc, dans la culture asiatique, celui qui va avoir la responsabilité de le diriger. En ce qui concerne leur oralisation, on peut dire, en schématisant un peu les choses, que chaque kanji a généralement deux lectures (parfois plus) : une lecture dite on'yomi, qui correspond à la lecture d'origine chinoise, souvent utilisée dans les mots composés de plusieurs kanji (comme dans le mot 会話 kaiwa) et une lecture dite kun'yomi, qui correspond à la lecture d'origine japonaise, souvent utilisée pour désigner le radical d'un verbe ou d'un adjectif. Dans le cas des verbes, le kanji est souvent combiné avec des hiragana (comme dans 話す hana-su, " parler ", où le kanji seul 話 se lit hana suivi de l'hiragana す su). On rencontre également, mais de manière beaucoup plus limitée, des lettres romaines souvent utilisées sous forme d'initiales pour désigner de manière abrégée des mots d'origine étrangère (comme OL pour Office Lady par exemple).

Pour mieux comprendre comment ces différents systèmes de signes s'articulent entre eux, il est important d'observer qu'à l'école primaire les hiragana, puis les katakana sont enseignés en premier, ce qui permet aux jeunes japonais de lire et d'écrire tous les mots qu'ils entendent. Les kanji, qui font l'objet d'un apprentissage beaucoup plus long s'étalant du début de l'école primaire à la fin des études secondaires, seront intégrés progressivement. Pendant la phase de découverte, une transcription en hiragana de petite taille au-dessus de chacun des kanji encore non assimilés, pourra être indiquée pour permettre à l'enfant d'en connaître la prononciation. C'est ce que l'on appelle les furigana dont l'utilisation constitue une étape intermédiaire entre la connaissance d'un mot écrit de manière phonétique (au moyen des hiragana) et celle d'un mot écrit de manière conceptuelle (au moyen des kanji). En effet, une fois que les kanji seront considérés comme connus, ils pourront être utilisés dans les textes étudiés sans furigana. D'autre part, lorsqu'ils s'expriment à l'écrit, les jeunes Japonais utilisent souvent les hiragana pour écrire des mots qu'ils sont censés avoir appris à écrire en kanji, mais qu'ils n'arrivent pas à retrouver. On remarquera d'ailleurs qu'avec la généralisation de l'utilisation des outils informatiques, les adultes rencontrent également cette difficulté lorsqu'ils sont en situation d'écriture manuscrite. Ces exemples montrent ainsi que les kanji ne sont pas des éléments absolument indispensables au processus d'encodage de l'écrit ; toutefois, si leur utilisation perdure, c'est qu'elle répond à d'autres besoins, que nous détaillerons plus loin.

Sur le plan de la structure linguistique, la langue japonaise est composée de quatre types de mots différents :

- les wago, qui sont les items lexicaux d'origine japonaise et qui se retranscrivent soit en hiragana soit avec des kanji utilisant des lectures kun'yomi (comme 話す hanasu par exemple) ;

- les kango, qui sont les items lexicaux d'origine chinoise et qui se retranscrivent avec des kanji utilisant des lectures on'yomi (comme 会話 kaiwa par exemple) ;

- les gairaigo, qui sont les items lexicaux d'origine étrangère qui se retranscrivent en katakana (commeトークショー tôkushô pour le mot anglais talk-show) ; 
- les items lexicaux hybrides qui mixent deux systèmes (wago et kango comme dans 話し中 hanashichû, " en discussion " ou kango et gairaigo comme dans 会話クラス kaiwa kurasu, "la classe de conversation").

Sur le plan quantitatif, d'après l'édition de 2002 du dictionnaire Shinsen Kokugoliten, les kango représenteraient $49,1 \%$ des entrées, contre $33,8 \%$ pour les wago, $8,8 \%$ pour les gairaigo et $8,4 \%$ pour les mots hybrides. Ceci montre la prédominance des kango, même si la tendance actuelle est à l'augmentation rapide de l'importance des gairaigo qui sont très utilisés dans tous les domaines où la culture étrangère, notamment occidentale, fait office de référence.

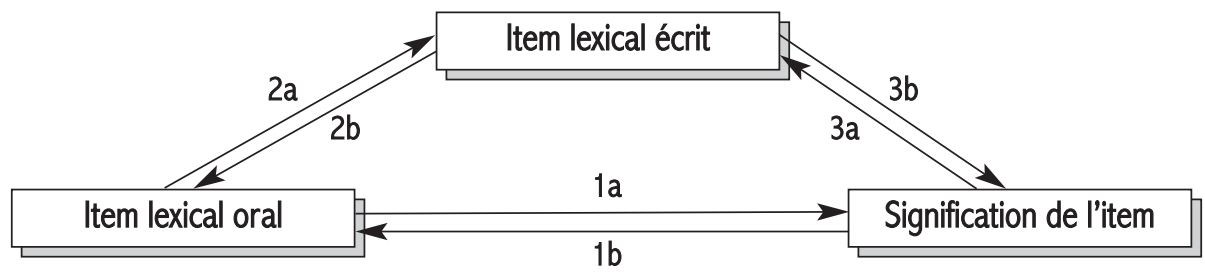

Schéma 1 : Les relations linguistiques entre item lexical et signification.

La structure de la langue induit donc des relations particulières entre " item lexical oral ", " item lexical écrit " et signification, l'» item lexical oral " étant défini comme la version oralisée de l'unité autonome constituante du lexique et l'u item lexical écrit " comme sa version écrite. Comme illustré sur le schéma ci-dessus, chacune de ces parties est reliée aux deux autres dans les deux directions. Nous avons tout d'abord la relation de l'item lexical oral à la signification (1a) mise en œuvre dans la compréhension orale ainsi que sa réciproque (1b) mise en œuvre dans l'expression orale. Puis, la relation de l'item lexical oral à l'item lexical écrit (2a) mise en œuvre dans la transcription écrite de messages oraux et sa réciproque (2b) mise en œuvre dans la lecture oralisée. Enfin la relation de l'item lexical écrit à la signification (3a) mise en œuvre dans la lecture non oralisée, surtout possible sur les associations de kanji présente dans les kango. On notera que c'est cette relation (3b), spécifique aux langues utilisant des "idéogrammes ", qui rend possible les stratégies spécifiques de compréhension et d'inférence. Sa réciproque (3a) semblerait a priori peu développée (si tant est qu'elle existe autrement qu'au plan théorique) : le procédé consisterait à encoder un concept directement à l'écrit, sans passer par la phase mentale d'oralisation.

\section{L'inférence directe et indirecte}

Mon propos étant d'étudier les mécanismes de l'inférence à l'écoute, nous allons nous intéresser plus précisément aux manières d'aller de l'item lexical oral à la signification, directement ou indirectement ; en effet, si l'inférence la plus largement identifiée dans toutes les langues 
consiste à inférer le sens d'un mot uniquement à partir du contexte, mécanisme que j'appellerai l'inférence directe (1a), j'ai pu constater, lors de ma propre pratique de l'écoute, l'existence d'un mécanisme d'inférence indirecte qui tente d'associer à un item oral entendu inconnu, une série d'items écrits (2a) sélectionnés à partir d'informations données par le contexte. De cette étape d'inférence se dégage une signification (3b). Tout se passe comme si, partant de l'oral, nous faisions un détour par l'écrit pour arriver à la signification.

- L'inférence peut être totale : pour un item lexical oral qui se décompose en plusieurs lexèmes (défini ici comme l'unité minimale de sens et de son qui compose un item lexical) correspondant généralement à plusieurs kanji dans l'item lexical écrit équivalent, l'inférence va porter sur la totalité de l'item oral, donc sur l'ensemble des lexèmes qui le constituent. Par exemple, j'entends l'item dôwa, que je ne connais pas, et je vais associer le premier lexème dô au kanji 童 connu, "l'enfant ", et le second wa au kanji connu 話, "la parole ", donc par association d'idées, je vais inférer que dôwa 童話 doit signifier " histoire pour enfants ". Mais l'inférence peut être également partielle. C'est le cas lorsque l'item inconnu comporte un lexème connu que je parviens immédiatement à repérer. En reprenant l'exemple ci-dessus, si je ne connais pas ou ne fais pas le rapprochement du lexème dô avec le kanji 童 signifiant " enfant ", je peux inférer partiellement le sens de l'item oralisé dôwa en le reliant au sens du second kanji, wa 話 qui est très fréquemment relié dans les kango au concept de " parole " ou d'" histoire " (kaiwa 会話, " la conversation ", denwa 電話, "le téléphone ", shinwa 神話, "le mythe ", etc.). Selon les cas, cette information va s'avérer suffisante ou non pour une bonne compréhension du message.

- Cependant, concernant l'inférence indirecte, on peut se demander s'il s'agit systématiquement d'un passage aux kanji et de leur représentation écrite qui permet la compréhension. En effet, il n'est pas impossible que certains lexèmes oraux (mono ou bi-syllabiques) soient associés dans l'esprit de l'auditeur à une signification du fait de leur apparition dans d'autres items lexicaux oraux. Par exemple, on peut avoir mémorisé le lexème wa de kaiwa ou de denwa comme étant porteur du sens de " parole " sans avoir forcément à l'esprit le kanji qui correspond. Pour plus de précision, on pourrait peut-être parler dans ce cas de lexèmes oraux signifiants qui auraient une polysémie élevée et seraient mémorisés comme des composants d'items lexicaux connus.

- L'inférence indirecte peut être plus ou moins précise ; en effet, lorsqu'il s'agit d'associer en japonais un lexème oral à son équivalent graphique (kanji dans la plupart des cas), l'éventail des possibilités peut être particulièrement large. Par exemple, dans un item lexical oral, les lexèmes qui se prononcent ka, ki, ken, kei, kô, shi, sei, shô, sô, tei, tô, hô, pour ne citer que les principaux, ont tous plus d'une vingtaine de transcriptions en kanji. Même si le contexte peut permettre de faire des choix, les hésitations restent possibles. Cette difficulté se double d'une polysémie particulièrement développée dans le registre lexical des mots d'origine chinoise, composés de deux ou trois kanji. Ainsi, l'interprète qui entendrait hors contexte le 
mot seikô pourrait lui associer, avec des kanji différents, une grande variété de significations comme "le succès " 成功, " la propension " 性向, "la conduite " 性行, "la précision " 精巧, " les relations sexuelles " 性交, "le programme politique " 政綱, " la fabrication de l'acier " 製鋼, " un style rigide "生硬, etc.

Une des difficultés qui guettent l'interprète occidental à l'écoute, outre celles directement liées à la polysémie, consiste à distinguer les voyelles courtes des voyelles longues. Dans le mode de transcription Hepburn, les voyelles prolongées sont marquées d'un trait horizontal souvent remplacé par un accent circonflexe ; on distingue par conséquent les lexèmes ko et kô qui renvoient chacun à une longue liste de kanji (respectivement vingt pour ko et soixante-six pour kô dans la liste des 2000 kanji usuels). Les possibilités d'inférence sont donc grandement augmentées par cette caractéristique du japonais qui constitue une grande partie de l'apprentissage de la langue dans les salles de cours. À ce propos, remarquons, que la polysémie peut gêner les Japonais eux-mêmes ; j'en veux pour preuve l'omniprésence de l'écrit à la télévision japonaise. Que ce soit dans les journaux télévisés mais également dans les programmes de variétés, l'écran du téléviseur est saturé de sous-titres qui transcrivent, de façon plus ou moins phatique, chaque parole prononcée. II n'est pas rare non plus lors d'une conversation entre Japonais, que l'un des interlocuteurs précise les kanji utilisés pour un mot quand il y a risque de confusion. On voit au travers de ces exemples que l'une des fonctions importantes des kanji dans la langue japonaise consiste à réduire la polysémie.

- Dans le cas de kango composés de trois kanji, l'inférence indirecte peut porter sur un item lexical déjà connu composé de deux kanji auxquels est associé un troisième qui joue un rôle de préfixe ou de suffixe. Par exemple, nous connaissons maintenant l'item kaiwa 会話, "la conversation ", mais nous ignorons encore l'item kaiwajutsu. Or nous connaissons le kanji 術 qui se lit jutsu en lecture on'yomi qui exprime l'idée de " technique " et de "savoir-faire". Nous allons donc en déduire que kaiwajutsu 会話術 peut signifier " la technique " ou "l'art de la conversation " et le contexte du message pourra ou non valider notre hypothèse. En introduisant des liens logiques dans la formation des mots, les kanji permettent d'alléger l'effort de mémoire pour l'apprentissage lexical en général et pour l'assimilation de corpus lexicaux importants en particulier. C'est notamment le cas en anatomie où les étudiants japonais en médecine doivent mémoriser plus de 7000 termes lors de leur première année d'étude.

- II existe également en japonais un grand nombre de kango composés de quatre kanji, qui sont souvent des associations de kango de deux kanji chacun comme dans kihonkaiwa 基本会話 qui est obtenu par association de kihon 基本, "la base " et de kaiwa 会話, "la conversation ", donc pour " la conversation de base ". Pour ce type de kango, le mécanisme d'inférence indirecte peut être légèrement différent ; on peut connaître la signification de l'un d'eux et inférer l'autre de la même manière qu'avec un autre kango de deux kanji 
comme présenté plus haut, mais la proximité au sein du même item lexical d'un autre élément signifiant permet une inférence plus pertinente. D'autre part, on peut aussi connaître séparément les deux kango constitutifs d'un kango de quatre kanji et ne pas comprendre la signification de ce nouvel item lexical, car toutes les associations ont un coût cognitif parfois trop important pour être effectuées en situation d'écoute. C'est le cas par exemple pour ipputasai一夫多妻, composé de ippu一夫 littéralement " un mari ", tasai 多妻 "plusieurs femmes", qui signifie "la polygamie".

- Parallèlement au processus d'inférence indirecte pour les items lexicaux inconnus, l'observation permet de remarquer qu'il existe également un processus de réactivation de la mémoire des items lexicaux partiellement oubliés ou qui ne sont connus de l'auditeur que dans la relation allant de l'item écrit à l'item oral (2b). Dans une activité de lecture, je sais oraliser le mot 会話 et j'en comprends la signification. En revanche, à l'écoute, je ne vais pas identifier directement l'item lexical kaiwa comme étant porteur de cette signification. Pour retrouver sa signification, je vais repasser par l'item écrit, avec moins d'hésitations toutefois que pour un item complètement inconnu, l'item ayant déjà été rencontré. On peut supposer que ce cas de figure est très fréquent chez les japonisants dont l'apprentissage est très axé sur l'écrit.

\section{Expérimentation sur l'inférence à l'écoute}

Afin d'étudier plus précisément l'importance de ces mécanismes d'inférence dans la compréhension de supports oraux en japonais pour un auditeur occidental, je me suis livré à l'expérience suivante. Après avoir sélectionné dix enregistrements d'émissions radiophoniques, d'une durée de 25 minutes chacun, portant sur des sujets scientifiques variés ne relevant pas particulièrement de mes domaines de spécialité, j'ai inventorié tous les items lexicaux qui me posaient problème, soit parce qu'ils m'étaient totalement inconnus, soit parce qu'ils étaient réactivés par cette écoute. Je les ai ensuite classés en six catégories :

- les items pour lesquels aucune inférence n'est possible (noté I0),

- les items inconnus pour lesquels il aura été fait une inférence directe de découverte (noté I1D) qui sera validée par la vérification,

- les items déjà rencontrés pour lesquels il aura été fait une inférence directe de réactivation (noté I1R) qui sera validée par la vérification,

- les items pour lesquels il aura été fait une inférence indirecte de découverte (noté 12D) qui sera validée par la vérification,

- les items déjà rencontrés pour lesquels il aura été fait une inférence indirecte de réactivation (noté $12 \mathrm{R}$ ) qui sera validée par la vérification,

- les items pour lesquels l'inférence directe ou indirecte s'est révélée erronée. 
Les résultats de cette expérimentation sont présentés dans le tableau ci-dessous :

\begin{tabular}{|c|c|c|c|c|c|c|c|}
\hline Thèmes des émissions & 10 & Er. & I1D & I1R & $12 \mathrm{D}$ & $12 \mathrm{R}$ & $\begin{array}{c}\text { Nombre total } \\
\text { d'items }\end{array}$ \\
\hline $\begin{array}{l}\text { La technique de la course } \\
\text { à pied (P1) }\end{array}$ & 12 & 1 & 5 & 3 & 9 & 13 & 43 \\
\hline $\begin{array}{l}\text { Les failles de la mémoire } \\
\text { (P2) }\end{array}$ & 4 & 2 & 5 & 3 & 9 & 18 & 41 \\
\hline $\begin{array}{l}\text { Le phénomène social } \\
\text { des « otaku » (P3) }\end{array}$ & 4 & 2 & 7 & 4 & 11 & 8 & 36 \\
\hline $\begin{array}{l}\text { Solliciter le cerveau } \\
\text { par l'écoute (P4) }\end{array}$ & 3 & 3 & 7 & 2 & 8 & 9 & 32 \\
\hline $\begin{array}{l}\text { Le fonctionnement } \\
\text { de l'épiderme (P5) }\end{array}$ & 14 & 3 & 9 & 2 & 19 & 8 & 55 \\
\hline $\begin{array}{l}\text { Les conditions carcérales } \\
\text { des étrangers }(\mathrm{P} 6)\end{array}$ & 6 & 8 & 9 & 3 & 18 & 4 & 48 \\
\hline $\begin{array}{l}\text { Le traitement } \\
\text { de la dépression (P7) }\end{array}$ & 5 & 2 & 8 & 4 & 10 & 3 & 32 \\
\hline La vie des gorilles (P8) & 10 & 6 & 12 & 4 & 16 & 5 & 53 \\
\hline $\begin{array}{l}\text { La dissection des animaux } \\
\text { (P9) }\end{array}$ & 8 & 5 & 16 & 4 & 14 & 6 & 53 \\
\hline $\begin{array}{l}\text { L'enseignement } \\
\text { de l'anatomie (P10) }\end{array}$ & 8 & 5 & 4 & 7 & 20 & 6 & 50 \\
\hline Total & 75 & 37 & 82 & 36 & 134 & 80 & 443 \\
\hline Pourcentages & $17 \%$ & $8 \%$ & $19 \%$ & $8 \%$ & $30 \%$ & $18 \%$ & $100 \%$ \\
\hline
\end{tabular}

Tableau 1 : Comptage des différents types d'inférences lors de l'écoute d'émissions radiophoniques à caractère scientifique.

Au vu de ces résultats, nous pouvons faire les remarques suivantes :

Tout d'abord, nous pouvons constater l'importance des mécanismes d'inférence dans leur ensemble. Ainsi, si l'on regroupe inférence directe et indirecte, qu'elle soit de "découverte " ou de "réactivation ", le mécanisme s'applique à $75 \%$ des items lexicaux posant problème. Parallèlement, nous pouvons aussi constater la faible part des items pour laquelle aucune inférence n'est possible (10), puisqu'elle n'est en moyenne que de $17 \%$. Pour les émissions dont le contenu était le plus difficile à appréhender ( $\mathrm{P} 1$ " les techniques de la course à 
pied ", P5 « le fonctionnement de l'épiderme », P6 « les conditions carcérales des étrangers, P8 " la vie des gorilles "), ce pourcentage peut monter jusqu'à un maximum de $28 \%$. On peut donc d'ores et déjà dire que, dans le cas du japonais, l'inférence peut être un outil quantitativement performant pour compléter les connaissances lexicales de l'auditeur dans le cadre de la compréhension orale.

- Un des résultats les plus étonnants de cette expérience est le faible nombre d'erreurs enregistrées (Er.), puisqu'il n'est que de $8 \%$ en moyenne (17\% en valeur maximale dans P6 : "les conditions carcérales des étrangers "). Pour les émissions dont les contenus ont été ressentis comme plus complexes (P1, P5, P6, P8), le nombre d'inférences erronées reste très faible. II est souvent compensé par un nombre plus important d'items lexicaux pour lesquels aucune inférence n'a été possible (10), de sorte que le total $10+$ Er reste relativement stable. Un kango pouvant avoir un nombre considérable de possibilités d'inférences, on ne peut que s'étonner de ce résultat. Pour un item qui se prononcerait kôsei que nous pouvons décomposer en deux lexèmes kô et sei, si nous nous référons à la liste des 2000 kanji usuels, nous avons soixante-six kanji possibles en lecture on'yomi pour kô et trentequatre pour sei, ce qui mathématiquement offre un total théorique de 2244 possibilités (pour environ une douzaine de combinaisons couramment utilisées). Même si le contexte permet de réduire considérablement le champ des possibilités, comment se fait-il qu'il n'y ait pas plus d'erreurs ? Nous verrons plus loin dans l'analyse d'exemples précis comment les différents types d'inférence se combinent pour arriver à ce résultat.

Si l'on compare cette fois les résultats enregistrés pour les deux formes d'inférence, on constate une nette supériorité de l'inférence indirecte sur l'inférence directe. En effet, si l'on exclut les inférences nulles (I0) et les inférences erronées (Er.), on obtient $65 \%$ d'inférences indirectes validées $(I 2 D+I 2 R)$, pour $35 \%$ d'inférences directes validées (I1D + I1R). Dans le même temps, si l'on observe de plus près les résultats obtenus pour les contenus perçus comme plus complexes (P1, P5, P6, P8), on voit que les valeurs enregistrées pour l'inférence indirecte de découverte (I2D) sont généralement supérieures à la moyenne (respectivement $21 \%$ pour $\mathrm{P} 1,35 \%$ pour $\mathrm{P} 5,38 \%$ pour $\mathrm{P} 6$ et $30 \%$ pour $\mathrm{P} 8$ alors que la moyenne est de $30 \%$ ). On pourrait donc légitimement émettre l'hypothèse que plus la compréhension du contenu est perçue comme difficile, plus l'auditeur va s'appuyer sur l'inférence indirecte pour surmonter ses difficultés de compréhension. Si l'on considère que par rapport à l'inférence directe, qui ne s'appuie que sur le contexte, l'inférence indirecte dispose d'un moyen supplémentaire pour accéder à la signification (le passage par l'item écrit), on peut comprendre pourquoi celle-ci peut s'avérer plus performante.

- Un rapide comptage des mots compris en inférence indirecte montre qu'il s'agit pour une écrasante majorité de kango généralement composés de deux kanji en lecture on'yomi, alors que les wago, à l'exception des mots composés (comme hanashiau 話し合う, " discuter ") sont plus souvent inférés sur le mode direct. 


\section{Analyse de cas d'inférence}

Les exemples présentés ci-dessous permettent de décrire plus en détail les différentes étapes du calcul inférentiel et d'en montrer quelques variantes liées à la fois à ma connaissance de l'item concerné et à sa structure linguistique.

Ex $n^{\circ} 1$ : Cas de l'item lexical oral shohan dans le contexte de l'univers carcéral :

1) Je décompose l'item shohan en deux lexèmes sho-han;

2) Grâce au contexte, j'associe le second lexème au han de hanzai, "le crime " (sans avoir eu recours au kanji 犯 ) ;

3) Ensuite, j'associe le premier lexème sho au kanji 初 qui exprime l'idée de " début " ;

4) Par association des deux éléments, j'en déduis que shohan doit signifier « le premier crime ";

5) Pour vérification, je recherche l'item lexical écrit complet de shohan qui est 初犯 et qui signifie bien "le premier crime".

Ex $n^{\circ} 2$ : Cas de l'item lexical oral giteisho dans le contexte de la protection de l'environnement :

1) Je décompose l'item giteisho en trois lexèmes gi-tei-sho;

2) Le contexte et la proximité dans la phrase du mot Kyoto me permettent de faire I'hypothèse qu'il s'agit du traité de Kyoto ;

3) Fort de cette hypothèse, j'associe le premier lexème gi au kanji 議 qui exprime l'idée de "délibération ";

4) Ensuite, j'associe le dernier lexème sho au kanji 書 qui exprime l'idée d'" écrit " ;

5) J'associe enfin, avec un degré de certitude moindre, le second lexème tei au kanji 定 qui exprime l'idée de "fixer ";

6) Je vérifie mon hypothèse de départ en recomposant l'item écrit complet giteisho 議定書, qui signifie bien « traité ".

Ex $n^{\circ} 3$ : Cas de l'item lexical oral taishinsekkei dans le contexte du recyclage des matériaux de construction :

1) Je décompose l'item taishinsekkei en deux items (de type kango), taishin et sekkei ;

2) J'identifie sekkei, item connu et y associe la signification de " plan " (sans y associer les kanji correspondants 設計) ;

3) Je décompose taishin, item inconnu en deux lexèmes, tai et shin ;

4) J'associe le second lexème shin au shin de l'item connu jishin, "le tremblement de terre " (sans avoir recours aux kanji correspondants 地震) ; 
5) J'en déduis directement la signification de l'item initial taishinsekkei comme étant " conception antisismique ";

6) Je recherche un kanji correspondant au premier lexème tai ayant le sens de " résistance " ou d' " opposition " et pense à 対 de tairitsu 対立, "l'opposition " (la vérification ultérieure montrera que cette dernière association était erronée, l'item écrit exact correspondant à taishinsekkei, " conception antisismique ", étant 耐震設計et non 対震設計).

- Lors de la décomposition de l'item lexical oral en lexèmes, il est à remarquer que si celle-ci peut paraître évidente pour l'auditeur chevronné, elle n'en est pas moins une source d'erreur potentielle. Par exemple, dans seishinkai, "le psychiatre ", il ne faut pas décomposer en trois lexèmes sei-shin-kai (kai étant un lexème très courant), mais en quatre sei-shin-ka-i pour faire une inférence correcte sur ce terme.

- On pourra noter qu'au sein même du processus d'inférence portant sur un seul item lexical, les stratégies mises en œuvre sont multiples et peuvent se combiner entre elles : inférence liée au contexte, inférence liée aux lexèmes écrits correspondants, inférence liée aux lexèmes connus dans d'autres items. À chaque étape de ce processus, l'auditeur a le choix entre l'une de ces différentes stratégies.

- Si, comme on l'a vu plus haut, les possibilités d'inférence sont élevées pour un item lexical donné sur un plan purement mathématique, on voit mieux maintenant comment le processus par étapes permet de réduire considérablement le nombre des significations possibles.

Il convient maintenant de préciser les limites de cette expérimentation et donc des conclusions que l'on peut en tirer.

- La restriction la plus importante est due au fait que cette expérience n'a été réalisée que sur une seule personne, à savoir moi-même, et l'on peut donc penser que le niveau de connaissance de la langue (connaissance lexicale, maitrise des kanji, etc.) influe sur les résultats observés et donc sur les conclusions qui en ont été tirées. II serait nécessaire d'élargir le panel à d'autres auditeurs ayant un niveau de langue différent, afin de vérifier si l'inférence varie en fonction des individus et des critères qui leur sont propres. Toutefois, les auditeurs doivent avoir, à mon sens, une solide connaissance des kanji usuels. De plus, le niveau de difficulté lexicale des enregistrements à étudier devrait se situer à la limite des compétences lexicales des auditeurs de manière à permettre une bonne compréhension globale, tout en laissant des " zones d'ombre " pour pouvoir mesurer leur capacité à inférer.

- Dans le même ordre d'idée, on pourrait faire valoir le fait que la conscience de l'existence de ces mécanismes influe sur la fréquence et l'efficacité de leur utilisation. Un auditeur qui n'aurait pas conscience de ces mécanismes aurait sans doute moins tendance à utiliser 
l'inférence indirecte que quelqu'un qui a constaté son efficacité : si, de façon générale, la confiance en ses propres capacités augmente les performances, on peut supposer que le domaine de l'inférence n'échappe pas à cette règle.

Le principe d'une bonne écoute en situation d'interprétation est de se concentrer directement sur la signification des messages et non sur les éléments qui les composent. Par contre, pour les besoins de cette expérience, c'est une écoute différente qui a été pratiquée ; en effet, la nécessité de noter les items lexicaux inconnus et les types d'inférence mis en œuvre a obligé parfois à interrompre l'enregistrement et à écouter plusieurs fois les mêmes passages, ce qui, en plus d'alourdir la démarche, nous éloigne encore un peu plus de l'écoute en situation d'interprétation qui, par définition, est continue et unique.

Pour aller plus loin dans l'analyse de l'inférence indirecte, on aurait pu affiner la grille d'observation en précisant par exemple les inférences indirectes totales et partielles, le degré de précision de l'inférence, les inférences indirectes erronées, etc.

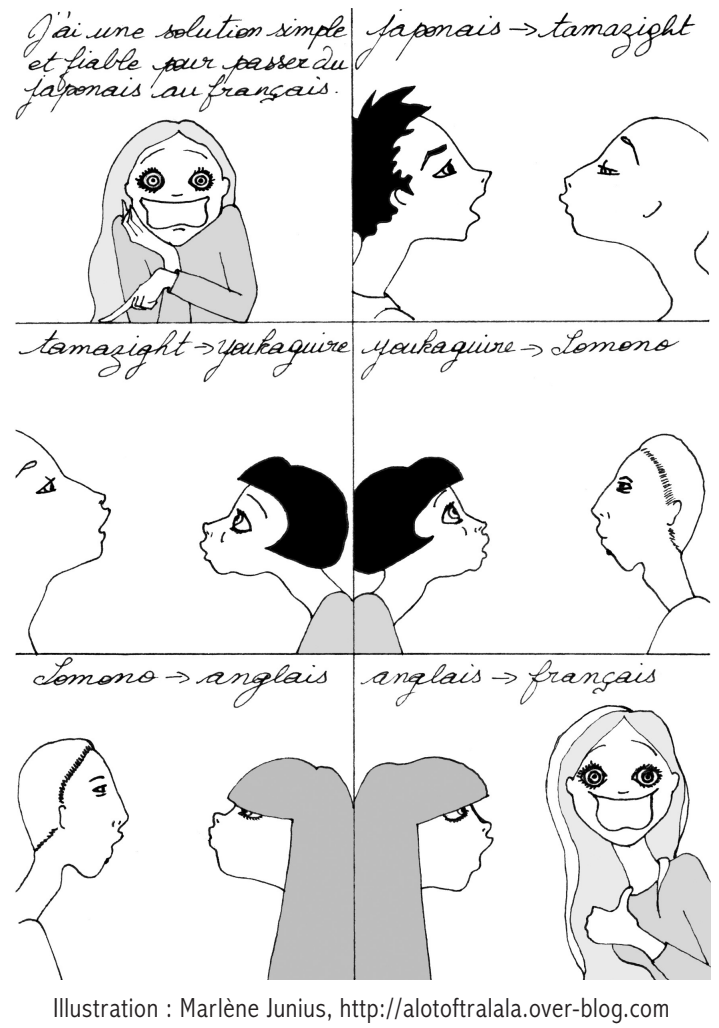




\section{Bilan et perspectives}

À la suite de cette expérience et des pistes de réflexion qu'elle suggère, je voudrais présenter ci-dessous les conséquences que j'en ai personnellement tirées sur ma pratique et mon entraînement à l'écoute. On pourra éventuellement y voir des directions didactiques destinées à de futurs interprètes ainsi qu'à tous les étudiants en japonais désireux d'améliorer leurs performances dans ce domaine.

II me semble important dans un premier temps de prendre confiance en ses propres capacités d'inférence et de les considérer comme un savoir-faire à valoriser au même titre que l'apprentissage lexical. En effet, dans un contexte traditionnel d'apprentissage des langues, l'inférence est parfois perçue comme un pis-aller, un moyen de pallier une connaissance lexicale approximative qui s'opposerait à une connaissance lexicale sûre et inaltérable. Or, la pratique nous montre que cette connaissance peut être beaucoup plus fluctuante dans le temps qu'il n'y paraît. On peut bien maîtriser toutes les relations associatives d'un item lexical telles que représentées plus haut (cf. schéma 1) à un moment donné et se rendre compte, quelque temps plus tard, que certaines de ces relations sont moins facilement mobilisables au plan cognitif. Dans ce contexte, l'inférence peut avoir un rôle de réactivation des connaissances lexicales passives qui me paraît d'autant plus utile que le champ lexical est étendu. D'autre part, plutôt que de l'envisager négativement comme une lacune, il faudrait à mon sens valoriser la compréhension partielle ou approximative : cumulée aux autres indices perçus par l'auditeur, elle peut s'avérer extrêmement précieuse pour atteindre une compréhension globale du message.

Par ailleurs, nous avons vu plus haut le rôle prépondérant de l'inférence indirecte, qui nécessite un passage par l'item lexical écrit pour inférer le sens. Celle-ci est particulièrement active pour les kango se composant de plusieurs kanji en lecture on'yomi. Afin de développer ses capacités à l'inférence indirecte, on ne peut donc que conseiller un solide apprentissage des kanji usuels ainsi que des révisions régulières. Dans le cadre de cet apprentissage, tous les exercices destinés à renforcer la relation allant de l'item lexical oral à l'item lexical écrit (2a) sont à privilégier pour compenser le traditionnel déséquilibre tentant à favoriser la relation inverse (2b). Dans le même ordre d'idées, il peut être également opportun de faire le point régulièrement sur toutes les possibilités de transcrire en kanji un item oral ou un lexème oral puisque c'est cette gymnastique mentale que l'auditeur devra reproduire lors de l'écoute, mais en temps limité. Plus généralement, il apparaît que tout travail sur l'écoute gagnerait grandement à être mis en étroite relation avec un travail spécifique sur les items lexicaux écrits posant problème et les kanji qui les composent. Parallèlement, lors du processus d'inférence, le recours à des lexèmes faisant partie de mots connus (sans faire forcément appel au kanji écrit) incite à penser que l'association d'un " mot référence " à chaque kanji connu peut permettre de favoriser ce processus. De plus, dans l'objectif de travailler sur des corpus lexicaux étendus, développer ses capacités à l'inférence indirecte apparaît comme plus performant 
qu'un apprentissage lexical classique basé uniquement sur la mémorisation de listes de vocabulaire.

Parallèlement à l'entraînement de la capacité d'inférence indirecte, tout ce qui peut contribuer également au renforcement de la relation allant de l'item lexical écrit à l'item lexical oral (1b) est aussi à entretenir. En effet, tous les items lexicaux, et en particulier les kango (rencontrés en connaissance passive par exemple dans des exercices de lecture extensive), peuvent nourrir un corpus lexical passif, qui est sollicité lors du mécanisme d'inférence indirecte.

Comme nous l'avons vu tout au long de cet article, il est très probable que l'inférence, et en particulier l'inférence indirecte, joue un rôle prépondérant dans les mécanismes de la compréhension orale du japonais de niveau soutenu par les auditeurs occidentaux. Pour confirmer cette hypothèse, il semblerait souhaitable qu'une expérimentation plus approfondie soit menée, permettant de faire des observations sur un plus grand nombre de sujets, voire même avec d'autres langues utilisant des idéogrammes. Mais si cette hypothèse est confirmée, il pourrait être extrêmement fructueux par la suite d'explorer les relations entre inférence indirecte et enrichissement lexical, tant ce dernier paraît être un enjeu central pour atteindre un très bon niveau de compréhension à l'oral dans ces langues.

franck.tiret@concordia-conseil.fr

Franck Tiret est interprète et formateur. Diplômé de I'INALCO, section japonais, en 1990, il commence à pratiquer l'interprétation français-japonais à Paris en free-lance avant de devenir traducteur et interprète salarié à Tokyo en 1991. Par la suite, il exercera les responsabilités de directeur commercial de la filiale d'une marque de haute-couture à Tokyo puis de consultant spécialisé dans l'importation de produits français au Japon. De retour en France depuis le début des années 2000, il est actuellement interprète, traducteur et consultant commercial au sein de la société Concordia Conseil, qu'il a créée. Il est également, depuis 2008, chargé de cours à l'université Stendhal de Grenoble.

\section{Références :}

GILE D. (1988), "Observations sur l'enrichissement lexical dans la progression vers un japonais " langue passive " pour l'interprétation de conférence ", in Meta, vol. 33, 1988, p. 79-89, accessible sur http://www.erudit.org/revue/meta/1988/v33/n1/002915ar.html

GILE D. (1982), " Initiation à l'interprétation consécutive à l'Institut national des langues et des civilisations orientales à Paris ", in Meta, vol. 27, $n^{\circ}$ 3, p. 347-351. 